\title{
Influencia del residuo de mampostería en la resistencia de concretos autocompactantes al ataque por sulfato de sodio
}

\author{
YIMMY FERNANDo SILVA URREGo \\ Silvio Delvasto Arjona
}

\section{Resumen}

En este trabajo se presentan resultados de un estudio experimental sobre la resistencia al ataque externo de sulfato de sodio $\left(\mathrm{Na}_{2} \mathrm{SO}_{4}\right)$ en concretos autocompactantes (CACs) con residuo de mampostería (RM). Los CACs presentaban un contenido de agua constante de 202,5 kg/m $\mathrm{m}^{3}$ y diferentes volúmenes de RM (0\%, 25\% Y 50\%) como reemplazo parcial de cemento Portland (OPC) expuesto a una solución de sulfato de sodio al 5\%. Las propiedades en estado fresco como fluidez, capacidad de paso y resistencia a la segregación se evaluaron mediante el flujo de asentamiento, embudo en V y caja en L. En estado endurecido, la resistencia a la compresión y expansión fueron determinadas. Por otra parte, técnicas de difracción de rayos X (DRX), microscopia electrónica de barrido (MEB) y espectroscopia de Infrarrojo con transformada de Fourier (FTIR) fueron aplicadas en pastas para investigar los efectos de los sulfatos sobre la microestructura. Los resultados mostraron que todas las mezclas cumplen las propiedades en estado fresco, además se encontró que cuando los CACs son inmersos en la solución de sulfato de sodio, el RM puede mejorar la resistencia de los CACs al ataque por sulfatos en comparación con el CAC solo de OPC.

Palabras clave: Residuo de mampostería; Concreto autocompactante; Sulfatos; Expansión; Etringita.

1 Universidad del Valle. Cali, Colombia.

Autor de correspondencia: Silva Urrego, Y.F. (Yimmy Fernando): Cra 56 \# 17-37. Teléfono: 3163658858. Correo electónico:yimmy.silva@correounivalle.edu.co
Historia del artículo:

Artículo recibido: 11-VI-2019/ Aprobado: 15-I-2020

Disponible online: 15 de enero de 2020

Discusión abierta hasta septiembre de 2021 


\section{Influence of masonry residue on the resistance of self-compacting concrete to the sodium sulfate attack}

\section{Abstract}

In this paper are shown the results of an experimental study of self compacting concretes with residue of masonry about their resistance to external sulfate attack, they presented a constant content of water of $202,5 \mathrm{~kg} / \mathrm{m}^{3}$ and different volumes of RM $(0 \%, 25 \%$ Y 50\%) as a partial replacement of Portland cement (OPC) exposed to a sulfate sodium solution at $5 \%$. The properties in fresh state as fluidity, passing ability and resistance to segregation were evaluated through slump flow, V-funnel and L-box. In hard state, the compression strength and expansion were determinate. Besides, techniques of X-ray diffraction (XRD), scanning electron microscopy (SEM) and Fourier transform infrared spectroscopy (FTIR) were applied to pastes in order to investigate the effects of sulfates on the microstructure. The results showed that all mixes have the properties on fresh state. Also, it was found that when CACs are exposed in a sodium sulfate solution, the RM can improve the resistance of CACs to the sulfates attack comparing with CAC only of OPC.

Keywords: Residue of masonry; Self-compacting concrete; Sulfates; Expansion; Ettringite.

\section{Influência do resíduo de mampostería na resistência de concretos autocompactantes ao ataque por sulfato de sódio}

\section{Resumo}

No presente trabalho são apresentados os resultados experimentais sobre a resistência ao ataque extremo de sulfato de sódio $\left(\mathrm{Na}_{2} \mathrm{SO}_{4}\right)$ em Concreto autoadensável (CACs) com resíduo de mampostería (RM). Os CACa aprecetam um teor de agua constate de 202,5 kg/mg3 e diferentes volumes de RM (0,25 e 50 \%) como substituição parcial do cimentos Portland (OPC) exposto numa solução de sulfato de sódio 5\%. As propriedades em estados fresco como fluidez, capacidade de passo e resistência à segregação foram avaliadas utilizando fluxo de assentamento, funil em V e caixa em L. em estado endurecido a resistência a compressão e expansão foram determinadas. Também foi determinado o efeito dos sulfatos na microestrutura através de difração de raios X (DRX), microscopia eletrônica de varredura (MEV) e espetroscopia de infravermelho com transformada de Fourier (FTIR). Os resultados mostrarem que todas as mesclas cumpre com as propriedades em estado fresco, além disso foi encontrado que quando os CACs são imersos na solução de sulfato de sódio o RM podem melhorar a resistência dos CACs ao ataque por sulfatos em comparação com o CAC somente com cimento.

Palavras-chaves: Resíduo de alvenaria; Concreto autoadensável; Sulfatos; Expansão; Etringite.

\section{Introducción}

El concreto es el material más usado en la industria de la construcción (Majhi y Nayak, 2019; Zhang et al. 2019), por la versatilidad, rentabilidad y confiabilidad que presenta, lo que ha conllevado que en los últimos años la producción de cemento Portland haya alcanzado 4,1 mil millones de toneladas al año (Tang et al. 2019). La elaboración de este aglomerante demanda una gran cantidad de materias primas y consumo energético considerable, lo que produce emisiones de $\mathrm{CO}_{2}$ a la atmosfera (Bulatović et al. 2017). Por lo que la necesidad de encontrar materiales que puedan sustituir el cemento es evidente, ya que con ello se evitaría una gran cantidad de emisiones de dióxido de carbono por cada tonelada de cemento producida. Además de la problemática que causa la fabricación de cemento Portland, en la actualidad se genera una gran cantidad de residuos de construcción y demolición (RCD) debido a la excavación, construcción, demolición o mantenimiento de las obras 
civiles (Wong et al. 2018; Muduli y Mukharjee, 2019), los cuales son depositados en vertederos y como relleno en canteras donde ya no se extrae material, lo cual conduce a un aumento de costo por el transporte y eliminación (Bravo et al. 2015).

Los RCD consisten en su mayoría en residuos sólidos de tipo inertes como concreto, mortero, ladrillo, metal, cerámica, madera, plástico y vidrio, los cuales pueden ser reciclados (Islam et al. 2019). Los ladrillos o residuos de mamposteria son el segundo material más empleado en la construcción después del concreto (Wong et al. 2018; Gálvez-Martos et al. 2018), el cual hace parte de los RCD. Además, este residuo se genera también durante su fabricación (Ercikdi et al. 2015). Por ejemplo, en Brasil, la industria ladrillera genera una cantidad considerables de ladrillos no conformes (principalmente debido a la falta de homogeneidad de las materias primas, resultado en la aparición de grietas y roturas) (Schackow et al. 2015). Por otra parte, en países como China, en los últimos años, sus actividades de demolición y reconstrucción han generado una gran cantidad de residuos de ladrillos que alcanzan los 0,4 billones de toneladas anuales (Li et al. 2016). En India, la mayoría de sus edificaciones son construidas con ladrillos de arcilla, por lo que anualmente se fabrican 250 mil millones de ladrillos (Kulkarni y Rao, 2016), lo que generaría también residuos de este material. En la Unión Europea EU los ladrillos de arcilla constituyen el 30\% del total de los RCD y alcanzando hasta cantidades más altas en países como España, el cual es de 54\% (Silva et al. 2019). Los residuos de ladrillo o de mampostería de ladrillo de arcilla son considerados como puzolanas, por su capacidad de reaccionar con el $\mathrm{Ca}(\mathrm{OH})_{2}$ producido en las reacciones de hidratación del cemento Portland (Lin et al. 2010; Silva et al. 2019). Sin embargo, en estado crudo, las arcillas no presentan actividad puzolanica, por lo que deben ser sometidas a un tratamiento térmico para permitir a este material pasar de un estado cristalino a un estado más o menos amorfo mediante la deshidroxilación (Mohammed, 2017). Para lograr este fenómeno (evaporación del agua ligada químicamente en la arcilla), estas deben de someterse a temperaturas entre 500 ${ }^{\circ} \mathrm{C}$ y $900{ }^{\circ} \mathrm{C}$ donde se da la formación de metacaolín y materiales amorfos (Ercikdi et al. 2015; Schackow et al. 2015). Por estas características, el polvo de ladrillo de arcilla podría emplearse como un material cementiceo suplementario, lo cual traería beneficios ambientales derivado de la menor demanda de cemento Portland y reducción en la cantidad de desecho.

Ademas de la problemática generada por los RCD, en la actualidad los estándares de construcción han cambiado, por lo que las modificaciones en el concreto son oportunas (Gill y Siddique, 2018). El concreto autocompactante (CAC) se adapta muy bien a los requerimientos actuales, debido a sus propiedades en estado fresco principalmente, como la capacidad de paso, capacidad de llenado o deformabilidad y estabilidad (resistencia a la segregación) (Abd Elaty y Ghazy, 2018). Por otra parte, el rendimiento en estado endurecido de los concretos es de gran importancia, por lo que la durabilidad a largo plazo es un factor a tener en cuenta. La variación de las características mecánicas del concreto a lo largo del tiempo es una consecuencia de los ataques químicos, físicos y ambientales. Entre los ataques más comunes que afectan la resistencia a la compresión es el ataque por sulfatos (Liu et al. 2012).

$\mathrm{El}$ ataque por sulfatos causa daños significativos en los materiales cementiceos, debido a las reacciones que se producen con la pasta, generando la formación de la etringita expansiva $\left(3 \mathrm{CaO} \cdot \mathrm{Al}_{2} \mathrm{O}_{3} \cdot 2 \mathrm{CaSO}_{4} \cdot 31 \mathrm{H}_{2} \mathrm{O}\right)$ y yeso $\left(\mathrm{CaSO}_{4} \cdot 2 \mathrm{H}_{2} \mathrm{O}\right)$, y en algunos casos la formación de la taumasita $\left(\mathrm{CaSiO}_{3} \cdot \mathrm{CaCO}_{3} \cdot \mathrm{CaSO}_{4} \cdot 15 \mathrm{H}_{2} \mathrm{O}\right)$ la cual requiere de bajas temperaturas, humedad, iones carbonatos y fuente de silicato de calcio (Skaropoulou et al. 2013). La resistencia a sulfatos de los materiales a base de cemento depende de varios factores entre los que esta la estructura del poro, la disponibilidad de $\mathrm{C}_{3} \mathrm{~A}$ y $\mathrm{Ca}(\mathrm{OH})_{2}$ (Irbe et al., 2019). Esto puede mejorarse mediante el empleo de Clinker con un contenido inferior de $\mathrm{C}_{3} \mathrm{~A}$ o disminuyendo la cantidad de $\mathrm{C}_{3} \mathrm{~A}$ mediante el empleo de un material cementicio suplementario, ya que además de tener un efecto dilución en el $\mathrm{C}_{3} \mathrm{~A}$, puede consumir la portlandita $\left(\mathrm{Ca}(\mathrm{OH})_{2}\right)$.

El empleo de RCD como componente del concreto juega un papel importante actualmente en las actividades de ingeniería respecto al impacto ambiental y de sostenibilidad en la fabricación de este material compuesto. El uso del residuo de mampostería (RM) como reemplazo parcial del cemento Portland disminuye el impacto negativo y los costos en la elaboración de concreto autocompactante (CAC). Sin embargo estudios sobre la durabilidad de CAC con RM no han sido reali- 
zados. Por ello, el propósito de esta investigación fue evaluar el efecto del RM proveniente de la demolición y remodelación de viviendas sobre la resistencia de concretos autocompactantes expuesto a una solución de sulfato de sodio, durante un periodo de 360 días.

\section{Programa Experimental \\ 2.1. Materiales}

\subsubsection{Cemento Portland (OPC)}

El cemento Portland empleado en este estudio fue tipo Uso general-UG de acuerdo con las especificaciones de la NTC 121. La composición química del cemento se muestra en la Tabla 1, la cual fue obtenida mediante fluorescencia de rayos x (FRX).

\subsubsection{Residuo de mampostería (RM)}

El residuo de mampostería se obtuvo de remodelaciones y construcciones de viviendas familiares (Figura 1). Después de su muestreo, el RM se sometió a un proceso de conminación en un molino de bolas hasta obtener un tamaño de partícula impalpable, cercano al del OPC empleado.

\section{TABLA 1. COMPOSICIÓN QUÍMICA Y PROPIEDADES} FÍSICAS DEL OPC Y RM

\begin{tabular}{|c|c|c|}
\hline Composición & OPC (\%) & RM (\%) \\
\hline $\mathrm{CaO}$ & 55,66 & 7,88 \\
\hline $\mathrm{SiO}_{2}$ & 19,39 & 56,86 \\
\hline $\mathrm{Al}_{2} \mathrm{O}_{3}$ & 4,13 & 15,53 \\
\hline $\mathrm{Fe}_{2} \mathrm{O}_{3}$ & 4,70 & 7,63 \\
\hline $\mathrm{SO}_{3}$ & 3,9 & 0,55 \\
\hline $\mathrm{Na}_{2} \mathrm{O}$ & 0,31 & 2,49 \\
\hline $\mathrm{MgO}$ & 1,70 & 2,95 \\
\hline $\mathrm{K}_{2} \mathrm{O}$ & 0,28 & 1,36 \\
\hline $\mathrm{TiO}_{2}$ & 0,25 & 0,75 \\
\hline \multicolumn{3}{|c|}{ Propiedades Físicas } \\
\hline Gravedad especifica $\left(\mathrm{kg} / \mathrm{m}^{3}\right)$ & 3100 & 2630 \\
\hline Perdida por ignición (\%) & 9,21 & 3,39 \\
\hline $\begin{array}{l}\text { Tamaño medio de partícula } \\
(\mu \mathrm{m})\end{array}$ & 20,67 & 24,08 \\
\hline
\end{tabular}

Figura 1. Residuo de mampostería generado en construcción y remodelación de viviendas familiares

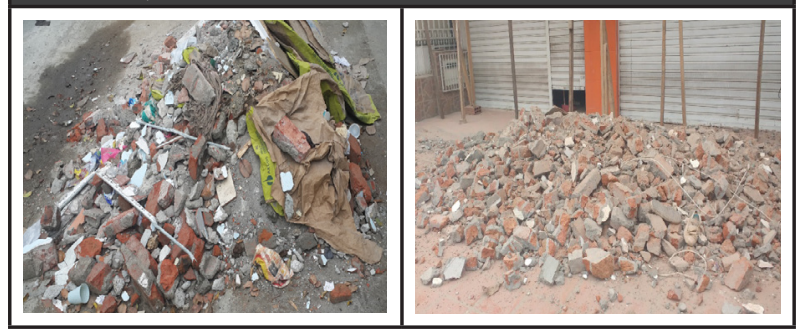

\subsubsection{Agua y solución de Sulfato de sodio}

Dos tipos de líquido se utilizaron en este estudio. Primero se empleó agua potable para la elaboración de los concretos y como ambiente de curado de referencia. El segundo, fue una solución de $50 \mathrm{~g} / \mathrm{L}$ de solución de $\mathrm{Na}_{2} \mathrm{SO}_{4}$ preparado según la norma ASTM C1012.

\subsubsection{Agregados}

El agregado fino empleado fue arena de rio con un módulo de finura de 2,26 y una gravedad especifica de $2.420 \mathrm{~kg} / \mathrm{m}^{3}$. Mientras que el agregado grueso fue grava triturada con un tamaño máximo de 12,7 mm y gravedad especifica de $2.780 \mathrm{~kg} / \mathrm{m}^{3}$. La absorción del agregado fino y grueso fue de $1,92 \%$ y $1,56 \%$ respectivamente.

\subsubsection{Suplerplastificante (SP)}

Un suplerplasficante Sikaplast- $328^{\circledR}$ se empleó para lograr la fluidez y trabajabilidad requerida en los CACs. Según la información del proveedor, el superplastificante presenta las siguientes propiedades: liquido de color café, densidad de $1080 \pm 0,02 \mathrm{~kg} / \mathrm{m}^{3}$ y un $\mathrm{pH}$ mínimo de 3,5.

\subsection{Proporciones de mezcla}

Tres mezclas en total se desarrollaron. Una fue la mezcla de control (PATRON), y otras dos mezclas (25\% RM y 50\% RM) fueron elaboradas reemplazando el $25 \%$ y $50 \%$ del cemento Portland por residuo de mampostería. El contenido de los agregados se fijó en $1581,32 \mathrm{~kg} / \mathrm{m}^{3}$ y se mantuvo constante el contenido de agua y de superplastificante en $202,5 \mathrm{~kg} / \mathrm{m}^{3}$ y $5,3 \mathrm{~kg} / \mathrm{m}^{3}$ respectivamente. Los diseños de mezclan se registraron en la Tabla 2. 
TABLA 2. DOSIFICACIÓN DE LOS CONCRETOS AUTOCOMPACTANTES CON RESIDUO DE MAMPOSTERÍA

\begin{tabular}{|c|c|c|c|c|c|c|}
\hline \multirow{2}{*}{ Mezcla } & \multicolumn{7}{|c|}{ Componentes de los CACs $\left(\mathrm{kg} / \mathrm{m}^{3}\right)$} \\
\cline { 2 - 8 } & OPC & RM & Agua & Arena & Grava. & SP \\
\hline & $\left(\mathrm{kg} / \mathrm{m}^{3}\right)$ & $\left(\mathrm{kg} / \mathrm{m}^{3}\right)$ & $\left(\mathrm{kg} / \mathrm{m}^{3}\right)$ & $\left(\mathrm{kg} / \mathrm{m}^{3}\right)$ & $\left(\mathrm{kg} / \mathrm{m}^{3}\right)$ & $\left(\mathrm{kg} / \mathrm{m}^{3}\right)$ \\
\hline PATRON & 500 & - & 202,5 & 895,11 & 686,21 & 5,3 \\
\hline $25 \%$ RM & 375 & 106,4 & 202,5 & 895,11 & 686,21 & 5,3 \\
\hline $50 \%$ RM & 250 & 212,8 & 202,5 & 895,11 & 686,21 & 5,3 \\
\hline
\end{tabular}

\subsection{Métodos de prueba}

\subsubsection{Evaluación de las propiedades en estado fresco de los CAC}

Las propiedades que caracterizan a los CAC se realizaron en las diferentes mezclas en estado fresco para determinar la capacidad de paso, capacidad de llenado y resistencia a la segregación. Estas pruebas se realizaron con los procedimientos recomendados por The SelfCompacting Concrete European Project Group (EPG, 2005).

Prueba de flujo de asentamiento (slump flow test). Este ensayo se realizó con el cono de Abrams., y determina la fluidez del concreto sin ninguna obstrucción. El diámetro del flujo se midió en dos direcciones perpendiculares una vez el flujo se había detenido. Esta prueba también permite realizar una inspección visual la cual brinda información sobre la resistencia a la segregación y uniformidad de la mezcla. Simultáneamente en esta prueba, se realizó el $\mathrm{T}_{500}$, el cual es el tiempo en el que el concreto alcanza un diámetro de $500 \mathrm{~mm}$, el cual indica la velocidad de flujo.

Prueba de embudo en V ( $V$-funnel test). En este ensayo se emplea un embudo en forma de V. Se llena con la mezcla y se deja fluir a través del embudo. Se toma el tiempo en que tarda el concreto fresco en salir de este equipo. Con este ensayo se determina la capacidad de llenado e indirectamente la viscosidad del CAC.

Caja en L ( L-box test). Este ensayo evalúa la capacidad de paso de los CAC en espacio confinado. Se realiza en un equipo con forma L, donde el compartimiento vertical se llena con el concreto, y luego se abre una compuerta para dejar fluir la mezcla a la parte horizontal. Una vez que se detiene el flujo del concreto, se mide la altura al final de la parte horizontal (H2) y la altura en la parte vertical (H1), y se calcula la relación de bloqueo (H2/H1), la cual indica la capacidad del concreto en pasar a través de los refuerzos; esta relación debe de estar entre 0,8 y 1 .

\subsubsection{Pruebas en estado endurecido}

Las pruebas en estado endurecido se llevaron a cabo para analizar la influencia del residuo de mampostería en los CACs. Se realizaron muestras cubicas de $50,8 \times 50,8 \times 50,8 \mathrm{~mm}$ para determinar la resistencia a la compresión de los CAC curados en agua y en la solución sulfato de sodio al 5\%, durante 28, 90, 180 y 360 días. Para cada uno de las edades mencionados, se ensayaron tres (3) cubos y se tomó el promedio de las lecturas. Este ensayo se realizó de acuerdo a la norma ASTM C39.

Para la prueba de expansión (cambio de longitud), se prepararon probetas de 25 × 25 × 285 mm, según la norma ASTM C1012. Las muestras se sumergieron en la solución de $\mathrm{Na}_{2} \mathrm{SO}_{4}$ al $5 \%$ para permitir que todas las superficies estuvieran en contacto con la solución agresiva durante 360 días, después de un curado durante 28 días en agua. Todos los contenedores se taparon para minimizar la carbonatación.

\subsubsection{Análisis microestructural}

El análisis microestructural se realizó en pastas (PATRON, 25\% RM y 50\% RM), las cuales fueron sometidas a las mismas condiciones que los CACs. Estas fueron analizadas por difracción de rayos X (DRX), espectroscopia de Infrarrojo con transformada de Fourier (FTIR) y microscopia electrónica de barrido (SEM).

\section{Resultados y discusiones}

\subsection{Propiedades en estado fresco}

La Tabla 3 presenta los resultados obtenidos de las mezclas de CACs en estado fresco, los cuales se encuentran en el rango especificado por The Self-Compacting Concrete European Project Group (EPG, 2005). 
TABLA 3. RESULTADOS DE LAS PROPIEDADES EN ESTAD FRESCO DE LOS CAC CON RM Y LOS CRITERIOS DE ACEPTACIÓN PARA LOS CAC

\begin{tabular}{|c|c|c|c|c|}
\hline \multirow{2}{*}{ MEZCLA } & Flujo de asentamiento & T500 & Embudo en $\mathrm{V}$ & Caja en $\mathrm{L}$ \\
\hline & $\mathrm{mm}$ & $s$ & s & \\
\hline PATRON & 680 & 3,64 & 9,26 & 0,85 \\
\hline $25 \% \mathrm{RM}$ & 690 & 3,39 & 9,35 & 0,9 \\
\hline $50 \% \mathrm{RM}$ & 700 & 3,19 & 9,21 & 0,91 \\
\hline \multicolumn{5}{|c|}{ Criterio de aceptación (EPG, 2005) [25]. } \\
\hline \multicolumn{2}{|c|}{ Método de prueba } & Unidades & mínimo & máximo \\
\hline \multicolumn{2}{|c|}{ flujo de asentamiento en cono de Abrams } & $\mathrm{mm}$ & 550 & 850 \\
\hline \multicolumn{2}{|c|}{ T500 (tiempo de flujo de asentamiento) } & segundos & $\leq 2$ & $>2$ \\
\hline \multicolumn{2}{|c|}{ Embudo en V } & segundos & $\leq 8$ & 25 \\
\hline \multicolumn{2}{|c|}{ Caja en $L$} & $\mathrm{H} 2 / \mathrm{H} 1$ & 0,8 & 1 \\
\hline
\end{tabular}

La prueba de flujo de asentamiento muestra que el residuo de mampostería no afecto de manera negativa el diámetro al final del ensayo. Todos los diámetros de flujo de las mezclas son más altos que el diámetro de flujo mínimo exigido, el cual es de $550 \mathrm{~mm}$; además, los CACs superan los $650 \mathrm{~mm}$ de diámetro en el flujo de asentamiento, el cual es recomendado en la práctica para garantizar una capacidad adecuada de autoconsolidación de los CAC (EFNARC, 2002). Por otra parte, EPG, clasifica estas mezclas como SF2, recomendándolas para muchas aplicaciones estructurales, tales como columnas, losas y vigas (EPG, 2005). En la prueba de $\mathrm{T}_{500}$ se obtuvieron tiempos en un rango entre 3,19 s y 3,64 $\mathrm{s}$, cumpliendo con las especificaciones de EPG. Un valor alto del $\mathrm{T}_{500}$ indica una mezcla con una viscosidad alta, que sería apropiada para concretos que se emplearían en obras con refuerzos o en secciones profundas, por otra parte un valor bajo en esta prueba, es apropiado cuando el concreto tiene que viajar por largas distancias horizontales sin mucha obstrucción (NRMCA, 2004).

Los resultados de la prueba de embudo en $\mathrm{V}$ reflejan la fluidez y viscosidad del CAC (Gülsan et al. 2019). La capacidad de llenado de acuerdo a este ensayo, los CAC se clasifican como VF2, ya que los tiempos obtenidos están entre 8 y 25 segundos, lo que puede suponer que estos concretos diseñados podrían ser apropiados para restringir la presión del encofrado y presentar una adecuada resistencia a la segregación (EFNARC, 2002).

La capacidad de paso se determinó en la prueba de caja en $\mathrm{L}$, donde se obtuvieron relaciones de altura que oscilaron entre 0,85 y 0,91 , lo que indica que todas las mezclas están dentro del rango especificado por la EPG (EPG, 2005). Se puede concluir de los resultados de la prueba de caja en $L$ que todas las mezclas tienen una adecuada capacidad de paso. La mayor capacidad de paso la presento la mezcla $50 \% \mathrm{RM}$.

En general, los resultados indican que el empleo de RM en la elaboración de CAC no efecta su desempeño en estado fresco, y satisface todos los requerimientos especificados en la EGP como son una adecuada fluidez y capacidad de paso.

\subsection{Propiedades en estado endurecido}

\subsubsection{Resistencia a la compresión}

\section{Curados en agua potable}

La Figura 2 muestra la resistencia a la compresión de los concretos autocompactantes curados en agua potable a diferentes días de curado, donde se observa un incremento a medida que aumentan los días de curado. La resistencia inicial (28 días de curado) la mezcla que presento la mayor resistencia fue el CAC PATRON, con un valor de 49,54 $\mathrm{MPa}$, siendo en un 21,7\% y 43,2\% más resistente que las mezclas 25\% y 50\% RM respectivamente, cabe destacar que en esta edad de curado se presenta la principal diferencia de resistencia entre el CAC PATRON y los CAC con RM. Esto se atribuye a que cuando se emplea una adición mineral por OPC, se puede producir diferentes efectos como son, el efecto dilución, efecto filler o la acción puzolanica (Bonavetti 
y Rahhal, 2006), en donde a esta edad de curado para los CAC con RM predomino el efecto dilución, lo que trae como consecuencia la disminución de la resistencia, a medida que aumenta el porcentaje de sustitución del cemento Portland. Sin embargo, 180 días después, la mezcla 25\% RM alcanza la resistencia desarrollada por el CAC PATRON, esto atribuido a la reacción puzolanica que presenta el RM, la cual es más notoria a los 360 días, donde el CAC con $25 \%$ de RM supera la resistencia del CAC de referencia en $3,5 \%$.

Figura 2. Resistencia a la compresión de CAC con RM curadas en agua

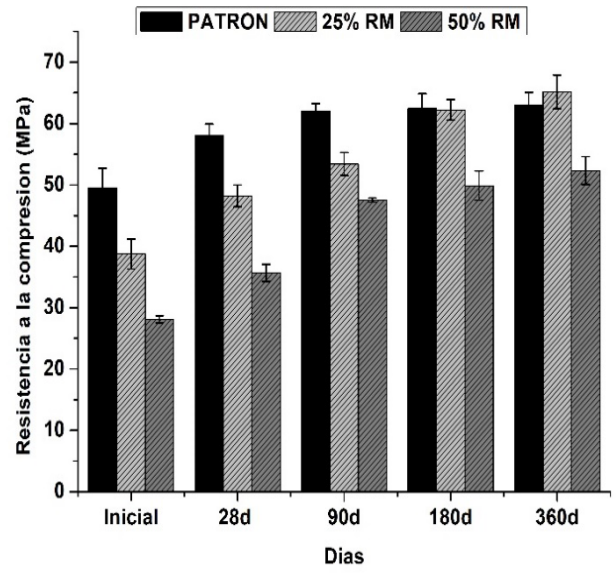

Expuesto a la solución al 5\% de $\mathrm{Na}_{2} \mathrm{SO}_{4}$

La Figura 3 muestra los resultados de resistencia a la compresión de los CACs inmersos en la solución de $\mathrm{Na}_{2} \mathrm{SO}_{4}$ al 5\%, donde la resistencia inicial indica la resistencia a la compresión justo antes de comenzar su periodo de exposición en esta solución, la cual corresponde a 28 días de curado en agua. A los 28 días de exposición se puede observar que todas las mezclas presentaron un aumento de la resistencia, esto atribuido a la formación de la etringita la cual al inicio de su formación tiene cierto impacto positivo en la resistencia, ya que aumenta la compacidad del concreto (Majhi y Nayak, 2019). A los 90 días de exposición, la mezcla PATRON presento una disminución de la resistencia de 7,62\% en comparación la resistencia presentada a los 28 días de exposición, esto debido a que la etringita empezó a tener un crecimiento excesivo dentro del concreto que puede tener efectos adversos, como la perdida de resistencia, sin embargo, la resistencia obtenida a esta edad, sigue siendo mayor a la presentada en el inicio del ensayo. A los 180 días y 360 días la resistencia de esta mezcla presento una pérdida significativa, esto atribuido a la expansión y agrietamiento producido por la etringita.

Los CAC con RM en ninguna de las edades de exposición presentaron caída de resistencia, lo que demuestra que el RM tuvo un comportamiento positivo frente al ataque del sulfato de sodio, debido a la menor disponibilidad de hidróxido de calcio debido al efecto dilución por el menor contenido de cemento además de la reacción puzolanica, que fija este compuesto.

Figura 3. Resistencia a la compresión de CAC con RM expuestas a una solución de $\mathrm{Na}_{2} \mathrm{SO}_{4}$

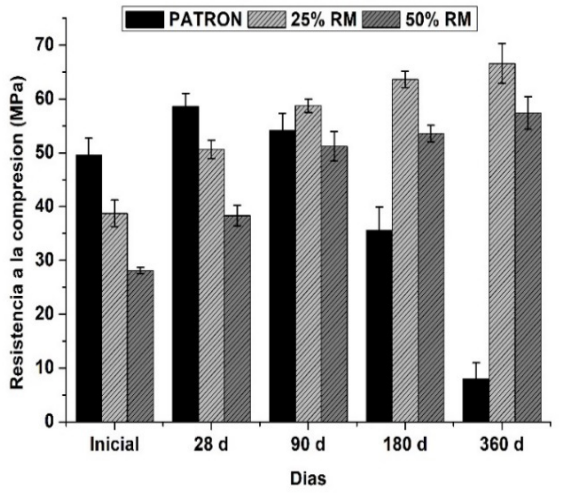

Figura 4. Cambio de la resistencia a la compresión de los CAC expuestos a la solución de $\mathrm{Na}_{2} \mathrm{SO}_{4}$ en relación con los curados en agua

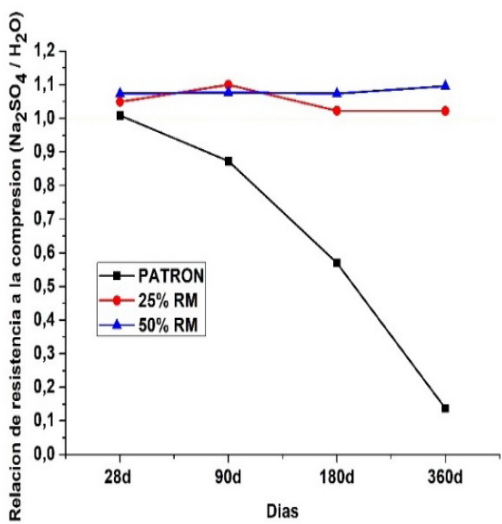


La Figura 4 muestra los valores relativos de la resistencia a la compresión. Los valores ilustrados son la relación entre la resistencia a la compresión de las concretos autocompactantes sumergidos en la solución $\mathrm{de}_{2} \mathrm{SO}_{4}$ y sus valores de referencia correspondiente a la misma edad curados en agua. Se puede concluir que el CAC PATRON fue el más afectado a la exposición en la solución de sulfato de sodio, mientras que las mezclas con RM presentaron un aumento de resistencia a la compresión en cada uno de los periodos de exposición evaluados respecto a sus correspondientes especímenes de referencia.

\subsubsection{Expansión}

La expansión de las barras de los CACs expuesta en la solución de $\mathrm{Na}_{2} \mathrm{SO}_{4}$ se presenta en la Figura 5. Se puede observar, para la mezcla PATRON, la expansión ocurre en dos etapas. Una primera etapa desde el inicio de la exposición hasta los 140 días, donde la expansión es muy baja, algunos autores la denominan periodo de inducción (Santhanam et al. 2002). Al final del periodo de inducción, inicia la etapa 2 , la cual es marcada por una expansión alta que continua a una velocidad elevada hasta que la probeta se desintegra, esto debido al aumento de la cantidad de etringita y yeso, la cual ya no puede acomodarse en la estructura del concreto.

El mecanismo de ataque que se presenta en la solución de $\mathrm{Na}_{2} \mathrm{SO}_{4}$, de acuerdo a Santhanam et al (2003) después del periodo durmiente (inducción), en la zona superficial de las barras de CAC se da la formación de etringita y yeso, la cual se comporta como una piel que trata de expandirse, cuando lo logra y empieza la generación de grietas en el interior del concreto, que con el tiempo la zona de la superficie empieza a deteriorarse debido a la penetración continua de la solución, la cual, al momento de alcanzar las zonas interiores agrietadas, reacciona con los productos de hidratación, conduciendo a la deposición de etringita yeso en las grietas y poros, debido a que estos lugares son los mejores sitios para la nucleación. En esta zona, al suceder esto empieza a ocurrir expansiones, causando fuerzas de tracción en la mezcla, lo que origina nuevas grietas en la zona interior. En esta instancia, en el concreto se presentan tres zonas distintas, la superficie desintegrada, la zona de deposición de los productos de ataque y la zona interior químicamente inalterada, cuando estas tres zonas están en el concreto el ataque progresa a un ritmo constante hasta que se produce la desintegración por completo (Santhanam et al. 2003).

Los CAC con RM, presentaron una reducción de la expansión drástica, ya que el empleo de adiciones activas disminuyen la permeabilidad de los CACs, lo que reduce la penetración de la solución de sulfato. Por otra parte, el RM es capaz de fijar la portlandita por la reacción puzolanica, lo que reduce la formación de yeso, lo que disminuye el efecto negativo del ataque de la solución de $\mathrm{Na}_{2} \mathrm{SO}_{4}$.

Figura 5. Expansión de CAC expuestos en la solución de $\mathrm{Na2SO} 4$ durante 365 días

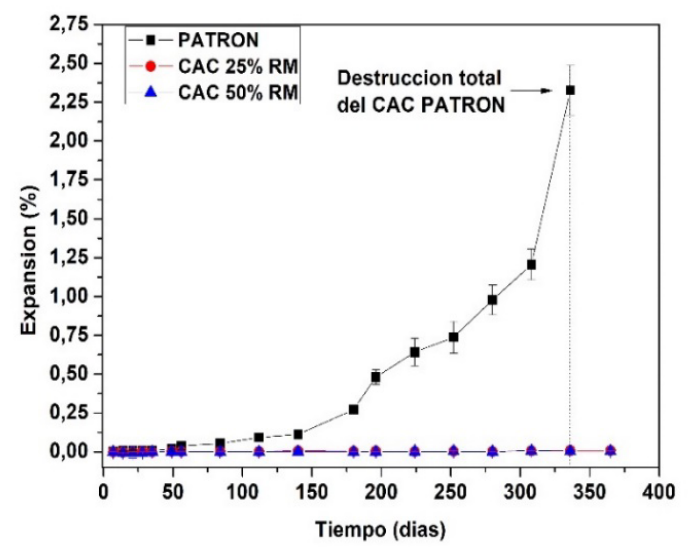

3.2.3. Microestructura (Micrografía Electrónical de barrido)

Las Figuras 6 y 7 muestran los productos microestructurales formados en las muestras curadas en agua y en la solución de sulfato de sodio mediante la técnica SEM. En la Figura 6 se aprecia las pastas a los 360 días de curado en agua, donde se puede observar una estructura compacta (magnificación x 1000), evidenciando una microestructura continúa, debido a un proceso de hidratación avanzado lo que conduce a una estructura compacta y densa (Sikandar et al. 2019). Por otra parte, a una magnificación mayor (x2000) se observa etringita ( $\mathrm{AFt}$ ) en forma de agujas y otros productos de hidratación incluido el silicato de calcio hidratado (C-S-H). 
Figura 6. Imágenes SEM de pastas curadas en agua por un periodo de 360 días

PATRON
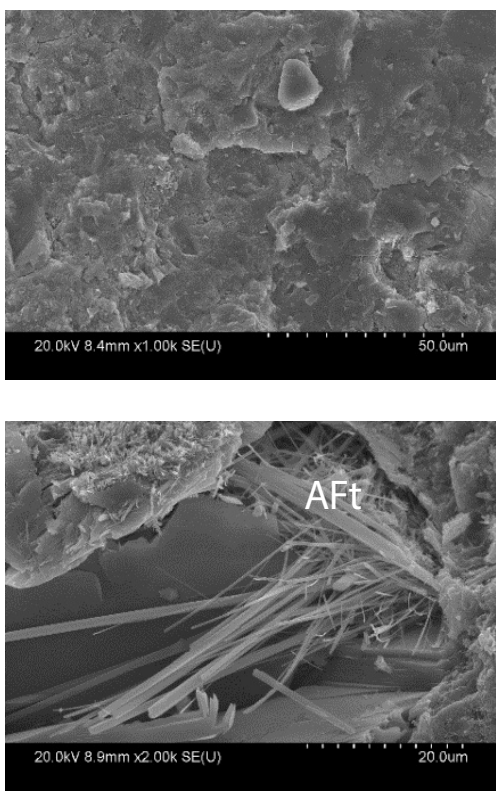

25\% RM
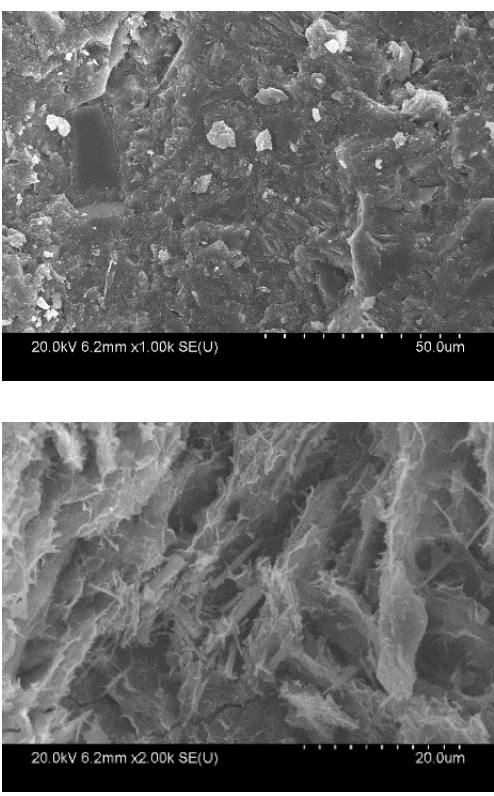

50\% RM
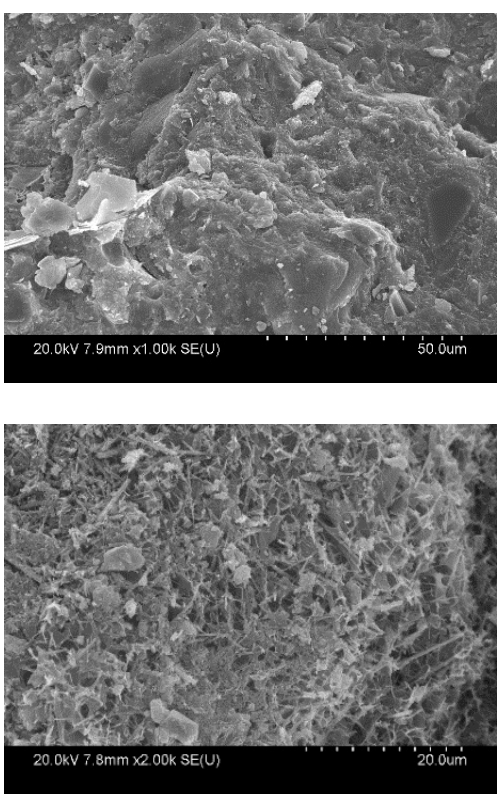

Figura 7. Imágenes SEM de pastas expuestas en $\mathrm{Na}_{2} \mathrm{SO}_{4}$ por un periodo de 360 días

\section{PATRON}
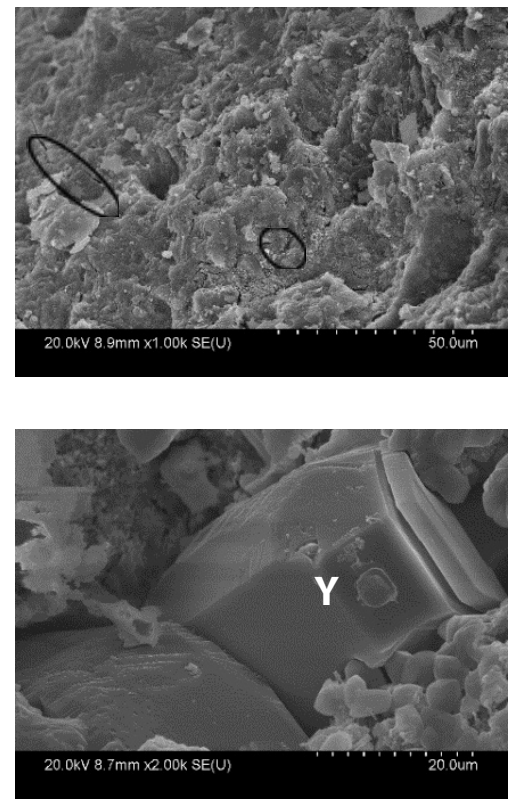

25\% RM
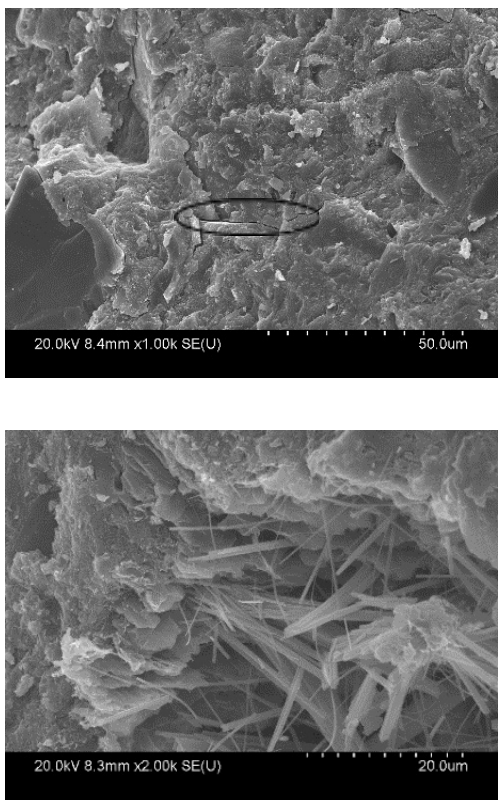

50\% RM
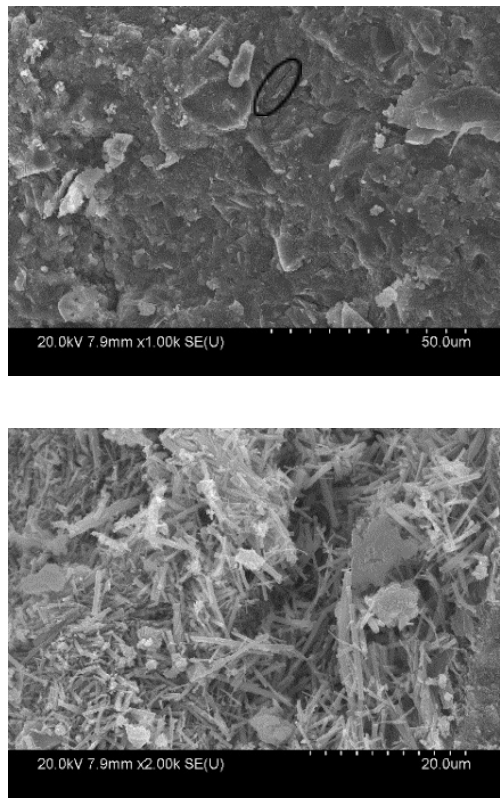
En la Figura 7 se observan los principales productos de deterioro como son el AFt y yeso (Y) $\left(\mathrm{CaSO}_{4} \cdot 2 \mathrm{H}_{2} \mathrm{O}\right)$ en las diferentes pastas, los cuales generan posteriormente expansión y agrietamiento (Liu et al. 2019). En las micrografías a una magnificación de x 1000 se puede apreciar pequeñas microgrietas que presentan las diferentes mezclas debido al ataque por los iones sulfatos. Al mismo tiempo, a una magnificación x 2000, los principales productos de la reacción química de la pasta con los iones sulfatos fueron láminas irregulares y cristales afilados en forma de aguja.

En comparación, con las muestras curadas en agua, hay pequeña manifestación de daños menores en las muestras con presencia de RM, indicando que estas mezclas exhibieron una insignificante incidencia la cual genero un hinchazón y agrietamiento mínimo, que por el contrario, en la mezcla PATRON se manifestó de gran manera.

\subsubsection{Caracterización de minerales por análisis de $D R X$}

Generalmente, el ataque de sulfatos a concretos se presenta cuando los iones sulfatos penetran la estructura de este material compuesto a través de los poros capilares y reaccionan con componentes (C3A, AFm no hidratado, alúmina reactiva de los MCS, portlandita etc) de la pasta de cemento y produce productos expansivos (etringita y yeso) (Li et al. 2019), los cuales generan agrietamiento y perdida de resistencia. Estos productos pueden ser identificados por DRX.

Para investigar los productos formados, las muestras en su totalidad se recolectaron, molieron y se analizaron por DRX. Los patrones de DRX de las mezclas PATRON, 25\% RM Y 50\% RM curadas en agua y expuestas en la solución de $\mathrm{Na}_{2} \mathrm{SO}_{4}$ se muestran en la Figura 8. Las fases de las pastas después de 360 días de exposición en la solución de sulfato de sodio son principalmente etringita (E), yeso (Y), cuarzo (Q) y calcita (C), lo que sugiere que si se ha presentado un ataque químico con sulfato (Chen et al. 2017). Además, es evidente que la intensidad de los picos de etringita y yeso es mayor en las muestras sumergidas en $\mathrm{Na}_{2} \mathrm{SO}_{4}$ que las curadas en agua para todas las mezclas, lo que está relacionado con la reacción química entre los iones sulfatos y los productos de hidratación del cemento Portland. Por otra parte, hay que destacar la menor intensidad del pico de portlandita en las mezclas expuestas en la solución de sulfato de sodio, lo que demuestra que la matriz de los diferentes CAC ha sufrido cierto deterioro por el ataque de sulfatos, ya que estos iones reaccionan con esta fase $\left(\mathrm{Ca}(\mathrm{OH})_{2}\right.$ para formar la etringita y el yeso $(\mathrm{Li}$ et al. 2019), conjuntamente con esta reacción, también se presenta en la reacción puzolanica y el efecto dilución en las mezclas con RM.

Figura 8. Patrón de DRX de las muestras curadas en agua y expuesta a la solución de $\mathrm{Na}_{2} \mathrm{SO}_{4}$ al $5 \%$, (a) PATRON, (b) $25 \%$ RM y (c) $50 \%$ RM
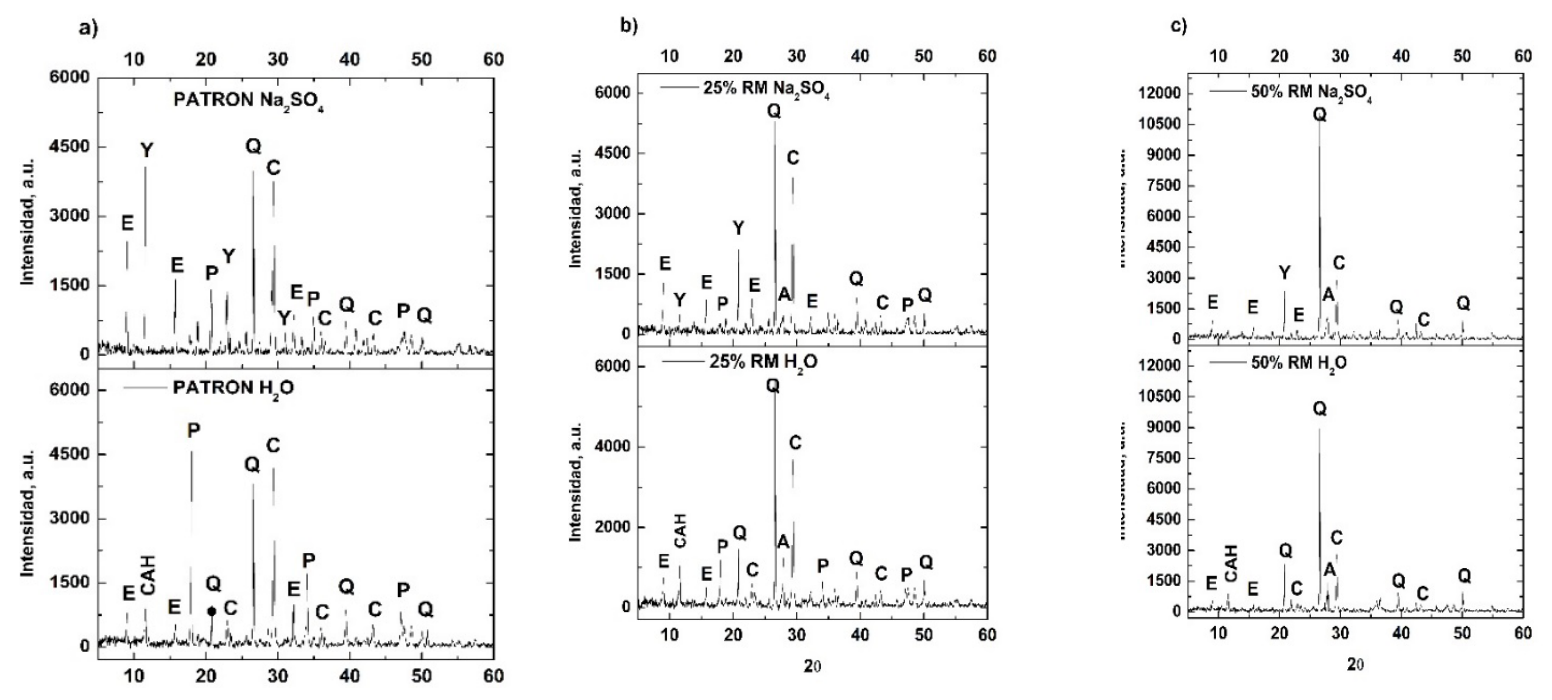


\subsubsection{Análisis FTIR}

La técnica FTIR se llevó a cabo para identificar los diferentes grupos funcionales presente en la pasta de los CACs. Como se observa en la Figura 9, las bandas 3445 $\mathrm{cm}^{-1}$ y $1649 \mathrm{~cm}^{-1}$ son causadas al estiramiento y flexión del grupo 0-H atribuido particularmente al yeso (Shaheen y Pradhan, 2017), la cual es más intensa para las pastas expuestas en la solución de $\mathrm{Na}_{2} \mathrm{SO}_{4}$ en comparación con las curadas en agua. Por otra parte, hay una banda pequeña del grupo $0-\mathrm{H}$ a $3663 \mathrm{~cm}^{-1}$ atribuida a la presencia de hidróxido de calcio de la pasta, la cual es más notoria para la muestra PATRON. La presencia de etringita como lo indica el DRX, fue corroborado por la presencia del pico Al-0 a $874 \mathrm{~cm}^{-1}$ en todas las mezclas (Shaheen y Pradhan, 2017), por otra parte, la banda a $1120 \mathrm{~cm}^{-1}$, la cual es más pronunciada en las mezclas expuesta a la solución de sulfato, sobre todo en la mezcla PATRON, es debido al estiramiento asimétrico de los enlaces $\mathrm{S}-\mathrm{O}$ en los grupos $\mathrm{SO}_{4}{ }^{-2}$, denotando también la presencia de etringita (Asensio de Lucas et al. 2016). Sin embargo, el yeso también muestra una banda de absorción en el rango entre 1100 a $1200 \mathrm{~cm}^{-1}$ correspondiente a las vibraciones $\mathrm{u} 3 \mathrm{del} \mathrm{SO}_{4}{ }^{-2}$ presente en este compuesto (Choudhary et al. 2015). Por otra parte, las bandas ubicadas entre $601 \mathrm{~cm}-1$ a 776 $\mathrm{cm}^{-1}$ también se asocian al yeso, las cuales son debido a las vibraciones v1 y v4 de $\mathrm{SO}_{4}^{-2}$ (Shaheen y Pradhan, 2017). La banda alrededor de $1430 \mathrm{~cm}^{-1}$ pertenece a la vibración de estiramiento típica de $\mathrm{C}-\mathrm{O}$, correspondiente al carbonato de calcio (Cai et al. 2018). La banda a 975 $\mathrm{cm}^{-1}$ a esta edad de hidratación corresponde a la precipitación del C-S-H amorfo.

Figura 9. Espectro FTIR de las mezclas PATRON, 25\% RM y $50 \%$ RM curadas en agua y expuesta a la solución de $\mathrm{Na}_{2} \mathrm{SO}_{4}$

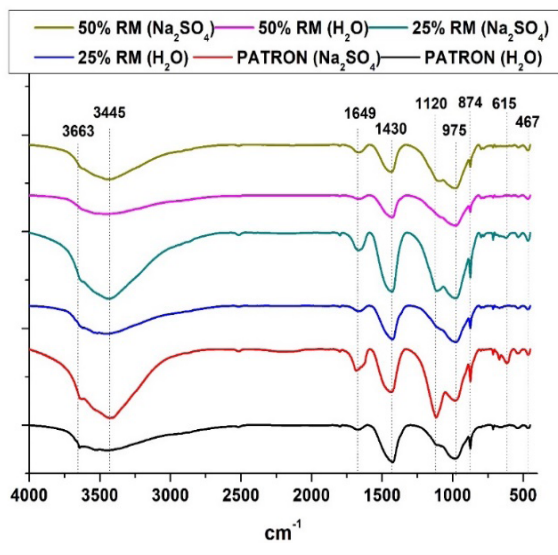

\section{Conclusiones}

Las conclusiones extraídas de esta investigación sobre el desempeño de concretos autocompactantes en estado fresco, y endurecido expuesto a sulfato de sodio se enumeran a continuación.

El empleo del residuo de mampostería en general mejora las propiedades de trabajabilidad de los concretos autocompactantes. Estas propiedades evaluadas mediante el flujo de asentamiento por cono de Abrams, embudo en $\mathrm{V}$ y caja en $\mathrm{L}$, en todos los CAC estuvieron en el rango adecuado para este tipo de concreto.

La resistencia a la compresión de los CACs se ve afectada por la presencia de RM, la cual es mas notoria a edades tempranas, y aún más para el concreto con $50 \%$ de RM. Sin embargo como la mayoría de materiales puzolanica (ceniza volante y escoria), la resistencia aumento a edades avanzadas, siento mayor la resistencia a la compresión para el CAC 25\% RM a los 360 días de curado en comparación al CAC PATRON.

La exposición de los concretos autocompactantes en la solución de sulfato de sodio genera en la matriz la formación de etringita y yeso, que proporciona una expansión interna conllevando al deterioro del concreto, sin embargo, es notorio que la formación de estas dos fases es menor en las mezclas con $25 \%$ y $50 \%$ RM, lo cual es evidente en la menor intensidad de los picos de etringita y yeso en los patrones de DRX en comparación con la mezcla con $100 \%$ cemento Portland (PATRON).

La adición del residuo de mampostería (RM) tiende a mejorar la resistencia al ataque por sulfato del concreto autocompactante, la cual es más notorio a edades prolongadas de exposición.

\section{Agradecimientos}

Los autores agradecen a la Universidad del Valle, especialmente a la Escuela de Ingeniería de los Materiales. Colciencias y al proyecto "Aerogeles silíceas obtenidas de la cascarilla de arroz mediante un proceso químico sostenible para aplicaciones en súper aislamiento térmico de edificaciones".

\section{Referencias}

Abd Elaty, M.A.A.; Ghazy M.F. (2018). Fluidity evaluation of fiber reinforced-self compacting concrete based 
on buoyancy law. HBRC Journal, 14, pp. 368-378. [Online] Disponible en: https://doi.org/10.1016/j. hbrcj.2017.04.003. [Consultado 2 de octubre de 2019].

Asensio de Lucas, E.; Medina, C.; Frías, M.; Sánchez de Rojas, M.I. (2016). Clay-based construction and demolition waste as a pozzolanic addition in blended cements. Effect on sulfate resistance. Construction and Building Materials, 127, pp. 950-058. [Online] Disponible en: https://doi.org/10.1016/j.conbuildmat.2016.10.047. [Consultado 9 de octubre de 2019].

Bonavetti, V.L.; Rahhal, V.F. (2006). Interacción de adiciones minerales en pastas de cemento. Revista de la Construccion, 52 (268), pp. 57-64. [Online] Disponible en: https://repositorio.uc.cl/handle/11534/11378 [Consultado 5 de octubre de 2019]

Bravo, M.; de Brito, J.; Pontes, J.; Evangelista, L. (2015). Mechanical performance of concrete made with aggregates from construction and demolition waste recycling plants. Journal of Cleaner Production, 99, pp. 59-74. [Online] Disponible en: https://doi. org/10.1016/j.jclepro.2015.03.012. [Consultado 1 de octubre de 2019]

Bulatović, V.; Melešev, M.; Radeka, M.; Radonjanin, V.; Lukić, I. (2019). Evaluation of sulfate resistance of concrete with recycled and natural aggregates, Construction and Building Materials. 152, pp. 614-631. [Online] Disponible en: http://dx.doi.org/10.1016/j.conbuildmat.2017.06.161. [Consultado 1 de octubre de 2019]

Cai, R.; He, Z.; Tang, S.; Wu, T.; Chen, E. (2018). The early hydration of metakaolin blended cements by non-contact impedance measurement. Cement and Concrete Composites, 92, pp. 70-81. [Online] Disponible en: https://doi.org/10.1016/j.cemconcomp.2018.06.001. [Consultado 10 de octubre de 2019].

Chen, F.; Gao, J.; Qi, B.; Shen, D. (2019). Deterioration mechanism of plain and blended cement mortars partially exposed to sulfate attack. Construction and Building Materials, 154, pp. 849-856. [Online] Disponible en: https://doi.org/10.1016/j.conbuildmat.2017.08.017. [Consultado 8 de octubre de 2019].

Choudhary, H.K.; A.V. A.; Kumar, R.; Panzi, M.E.; Matteppanavar, S.; Sherikar, B.N.; Sahoo, B. (2015). Observation of phase transformations in cement during hydratation. Construction and Building Materials,
101, pp. 122-129. [Online] Disponible en: https://doi. org/10.1016/j.conbuildmat.2015.10.027. [Consultado 10 de octubre de 2019].

EFNARC (2002). Specification and guidelines for selfcompacting concrete. European association for producers and applicators of specialist building products. [Online] Disponible en: http://www.efnarc.org/pdf/ SandGforSCC.PDF

EPG (2005). BIBM, CEMBUREAU, ERMCO, EFCA, EFNARC. The European guidelines for self compacting concrete: specification, production and use. The Self-Compacting Concrete European Project Group. [Online] Disponible en: http://www.efca.info/download/ european-guidelines-for-self-compacting-concretescc/. [Consultado 2 de octubre de 2019].

Ercikdi, B.; Külekci, G.; Yılmaz, T. (2015). Utilization of granulated marble wastes and waste bricks as mineral admixture in cemented paste backfill of sulphide-rich tailings. Construction and Building Materials, 93, pp. 573-583. [Online] Disponible en: http://dx.doi. org/10.1016/j.conbuildmat.2015.06.042. [Consultado 1 de octubre de 2019].

Gálvez-Martos, J.L.; Styles, D.; Schoenberger, H.; ZeschmarLahl, B. (2018). Construction and demolition waste best management practice in Europe. Resources, Conservation \& Recycling, 136, pp. 166-178. [Online] Disponible en: https://doi.org/10.1016/j.resconrec.2018.04.016. [Consultado 1 de octubre 2019].

Gill, A.S.; Siddique, R. (2018). Durability properties of self compacting concrete incorporating metakaolin and rice husk ash. Construction and Building Materials, 176, pp. 323-332. [Online] Disponible en: https://doi. org/10.1016/j.conbuildmat.2018.05.054. [Consultado 2 de octubre 2019]

Gülsan, M.E.; Alzeebaree, R.; Rasheed, A. A.; Nis, A.; Kurtoğlu, A.E. (2019). Development of fly ash/slag based self compacting geopolymer concrete using nano-silica and steel fiber. Construction and Building Materials, 211, pp. 271-283. [Online] Disponible en: https://doi.org/10.1016/j.conbuildmat.2019.03.228. [Consultado 5 de octubre de 2019].

Irbe, L.; Beddoe, R.E.; Heinz, D. (2019). The role of aluminium in C-A-S-H during sulfate attack on concrete. Cement and Concrete Research, 116, pp. 71-80. [On- 
line] Disponible en: https://doi.org/10.1016/j.cemconres.2018.11.012. [Consultado 2 de octubre 2019].

Islam, R.; Nazifa, T.H.; Yuniarto, A.; Uddin, A.S.M.S.; Salmiati, S.; Shahid, S. (2019). An empirical study of construction and demolition waste generation and implication of recycling. Waste Management, 95, pp. 10-21. [Online] Disponible en: https://doi.org/10.1016/j. wasman.2019.05.049. [Consultado 1 de octubre 2019].

Kulkarni, N.G.; Rao, A.B. (2016). Carbon footprint of solid clay bricks fired in clamps of India. Journal of Cleaner Production, 135, pp. 1396-1406. [Online] Disponible en: https://doi.org/10.1016/j.jclepro.2016.06.152. [Consultado 1 de octubre 2019]

Li, B.; Cao, R.; You, N.; Chen, C.; Zhang, Y. (2019). Products and properties of steam cured cement mortar containing lithium slag under partial immersion in sulfate solution. Construction and Building Materials, 220, pp. 596-606. [Online] Disponible en: https://doi. org/10.1016/j.conbuildmat.2019.06.062. [Consultado en 8 de octubre de 2019].

Li, H.; Dong, L.; Jiang, Z., Yang, X.; Yang, Z. (2016). Study on utilization of red brick waste powder in the production of cement-based red decorative plaster for walls. Journal of Cleaner Production, 133, pp. 1017- 1026. [Online] Disponible en: https://doi.org/10.1016/j. jclepro.2016.05.149. [Consultado 1 de octubre 2019].

Lin, K.L.; Chen, B.Y.; Chiou, C.S.; Cheng, A. (2010). Waste brick's potential for use as a pozzolan in blended Portland cement. Waste Management \& Research, 28, pp. 647-652. [Online] Disponible en: https:// DOI:10.1177/0734242X09355853. [Consultado 1 de octubre de 2019].

Liu, C.; Gao, J.; Chen, F.; Zhao, Y.; Chen, X.; He, Z. (2019). Coupled effect of relative humidity and temperature on the degradation of cement mortars partially exposed to sulfate attack. Construction and Building Materials, 216, pp. 93-100. [Online] Disponible en: https://doi.org/10.1016/j.conbuildmat.2019.05.001. [Consultado 8 de octubre de 2019].

Liu, T.; Teng, J.; Yan, G. (2012). The influence of sulfate attack on the dynamic properties of concrete column. Construction and Building Materials, 28, pp. 201-207. [Online] Disponible en: https://doi.org/10.1016/j. conbuildmat.2011.08.036. [Consultado 2 de octubre de 2019].

Majhi, R.K.; Nayak, A.N. (2019). Bond, durability and microstructural characteristics of ground granulated blast furnace slag baased recycled aggregate concrete. Construction and Building Materials, 212, pp. 578-595. [Online] Disponible en: https://doi.org/10.1016/j. conbuildmat.2019.04.017. [Consultado 1 de octubre de 2019].

Mohammed S. (2017). Processing, effect and reactivity assessment of artificial pozzolans obtained from clays and clay wastes: A review, Construction and Building Materials, 140, pp. 10-19. [Online] Disponible en: https://doi.org/10.1016/j.conbuildmat.2017.02.078. [Consultado 2 de octubre de 2019].

Muduli, R.; Mukharjee, B.B. (2019). Effect of incorporation of metakaolin and recycled coarse aggregate on properties of concrete, Journal of Cleaner Production. 209, pp. 398-414. [Online] Disponible en: https://doi. org/10.1016/j.jclepro.2018.10.221. [Consultado 1 de octubre de 2019].

NRMCA (2004). CIP 37 - Self Consolidating Concrete (SCC). Disponible en: https://www.nrmca.org/aboutconcrete/cips/37p.pdf

Santhanam, M.; Cohen, M.D.; Olek, J. (2002). Mechanism of sulfate attack: A fresh look Part 1: Summary of experimental results. Cement and Concrete Research, 32, pp. 915 - 921. [Online] Disponible en: https://doi. org/10.1016/S0008-8846(02)00724-X. [consultado 7 de octubre de 2019]

Santhanam, M.; Cohen, M.D.; Olek, J. (2003). Mechanism of sulfate attack: a fresh look Part 2. Proposed mechanisms. Cement and Concrete Research, 33, pp. 341 346. [Online] Disponible en: https://doi.org/10.1016/ S0008-8846(02)00958-4. [Consultado 7 de octubre de 2019].

Schackow, A.; Stringari, D.; Senff, L.; Correia, S.L.; Segadães, A.M. (2015). Influence of fired clay brick waste additions on the durability of mortars. Cement \& Concrete Composites, 62, pp. 82-89. [Online] Disponible en: http://dx.doi.org/10.1016/j.cemconcomp.2015.04.019. [Consultado 1 de octubre de 2019].

Shaheen, F.; Pradhan, B. (2017). Influence of sulfate ion and associated cation type on steel reinforcement corro- 
sion in concrete powder aqueous solution in the presence of chloride ions. Cement and Concrete Research, 91, pp. 73-86. [Online] Disponible en: https://doi. org/10.1016/j.cemconres.2016.10.008. [Consuldato 10 de octubre de 2019].

Sikandar, M.A.; Ahmad, W.; Khan, M.H.; Ali, F.; Waseem, M. Effect of water resistant $\mathrm{SiO}_{2}$ coated SrAl204: Eu2+ Dy3+ persistent luminescence phosphor on the properties of Portland cement pastes. Construction and Building Materials, 228, 116823. [Online] Disponible en: https://doi.org/10.1016/j.conbuildmat.2019.116823. [Consultado 8 de octubre de 2019].

Silva, G.; Castañeda, D.; Kim, S.; Castañeda, A.; Bertolotti, B.; Ortega-San-Martin, L.; Nakamatsu, J.; Aguilar, R. (2019). Analysis of the production conditions of geopolymer matrices from natural pozzolana and fired clay brick wastes. Construction and Building Materials, 215, pp. 633-643. [Online] Disponible en: https://doi.org/10.1016/j.conbuildmat.2019.04.247. [Consultado 1 de octubre de 2019].

Silva, Y.F.; Izquierdo, S.R.; Delvasto, S. (2019). Effect of masonry residue on the hydration of Portland cement paste. Revista DYNA, 86(209), pp. 367-377. [Online] Disponible en: http://doi.org/10.15446/dyna. v86n209.77286. [Consultado 1 de octubre de 2019].

Skaropoulou, A.; Sotiriadis, K.; Kakali, G.; Tsivilis, S. (2013). Use of mineral admixtures to improve the resistance of limestone cement concrete against thaumasite form of sulfate attack. Cement \& Concrete Composites, 36, pp. 267-275. [Online] Disponible en: https://doi. org/10.1016/j.cemconcomp.2013.01.007. [Consultado 2 de octubre de 2019].

Tang, Z.; Li, W.; Ke, G.; Zhou, J.L.; Tam, V.W.Y. (2019). Sulfate attack resistance of sustainable concrete incorporating various industrial solid waste. Journal of Cleaner Production, 218, pp. 810-822. [Online] Disponible en: https://doi.org/10.1016/j.jclepro.2019.01.337. [Consultado 1 de octubre de 2019].

Wong, C.L.; Mo, K.H.; Yap, S.P.; Alengaram, U.J. (2018). Potential use of brick waste as alternate concrete-making materials: A review. Journal of Cleaner Production, 195, pp. 226-239. [Online] Disponible en: https://doi. org/10.1016/j.jclepro.2018.05.193. [Consultado 1 de octubre de 2019]
Zhang Y.; Luo W.; Wang J.; Wang Y.; Xu Y.; Xiao J. (2019). A review of life cycle assessment of recycled aggregate concrete. Construction and Building Materials, 209, pp. 115-125. [Online] Disponible en: https://doi. org/10.1016/j.conbuildmat.2019.03.078. [Consultado 1 de octubre de 2019].

\section{PARA CITAR ESTE ARTÍCULO / TO REFERENCE THIS ARTICLE / PARA CITAR ESTE ARTIGO /}

Silva Urrego, Y.F.; Delvasto Arjona, S. (2020). Influencia del residuo de mampostería en la resistencia de concretos autocompactantes al ataque por sulfato de sodio. Revista EIA, 17(33) enero-junio, Reia33014 pág. 1-14. Disponible en: https://doi.org/10.24050/reia.v17i33.1361 